\title{
A Wavelet-Based Approach for Automatic Diagnosis of Strict Left Bundle Branch Block
}

\author{
Alba Martín-Yebra ${ }^{1,2}$, Juan Pablo Martínez ${ }^{2,1}$ \\ ${ }^{1}$ Centro de Investigación Biomédica en Red - Bioingeniería, Biomateriales y Nanomedicina, Spain \\ ${ }^{2}$ BSICoS Group, Aragón Institute of Engineering Research, IIS Aragón, Universidad de Zaragoza, \\ Zaragoza, Spain
}

\begin{abstract}
Cardiac resynchronization therapy $(C R T)$ is widely used in heart failure patients with left bundle branch block $(L B B B)$. However, the high false-positive rates obtained with the conventional $L B B B$ criteria limit the effectiveness of this therapy. This has yielded to the definition of a new stricter criteria for diagnosis. The aim of this work was to develop and assess a fully-automatic algorithm for strict LBBB diagnosis. Twelve-lead, high-resolution, 10-second ECGs from 602 patients enrolled in the MADIT-CRT trial were available. Data were labelled for strict $L B B B$ by 2 experts and divided into training $(n=300)$ and validation ( $n=302$, blind annotations to the investigators) sets for assessing algorithm performance. After QRS detection, a wavelet-based delineator was used to detect individual $Q$ $R-S$ waves, QRS onsets and ends, and identify the type of QRS pattern on each standard lead. Then, multilead QRS boundaries were determined in order to compute the QRS width. Finally, an automatic algorithm for notch/slur detection within the QRS complex was applied based on the same wavelet approach used for delineation. In the validation set, $L B B B$ was diagnosed with a sensitivity and specificity of $S e=92.9 \%$ and $S p=65 \%$ ( $A c c=79 \%, P P V=73.9 \%$ and $N P V=89.6 \%$ ). Results confirmed an accurate diagnosis of strict $L B B B$ based on a fully-automatic extraction of temporal and morphological QRS features.
\end{abstract}

\section{Introduction}

In the presence of left bundle branch block (LBBB), cardiac resynchronization therapy (CRT) has been postulated as the preferred option for re-synchronization of ventricular contraction in those failure hearts $[1,2]$. Consequence of the delayed left ventricular wall activation respect to the interventricular septum, LBBB manifests as a prolonged QRS duration and a threshold of $120 \mathrm{~ms}$ was set for conventional ECG diagnosis criteria. However, approximately one third of diagnosed patients was shown not to have complete LBBB $[3,4]$. The high false-positive rates obtained with those conventional LBBB criteria limit the ef- fectiveness of CRT in heart failure patients [4,5].

Later, simulation studies have evidenced the presence of mid-QRS notch or slurs in some leads in the presence of complete LBBB. A negative deflection in lead V1 or sex differences in QRS duration were also associated to LBBB. These observations yielded to the definition of a new set of stricter criteria for LBBB diagnosis [5]. It requires three conditions: $\mathrm{C} 1$ ) prolonged $\mathrm{QRS}$ duration ( $\geq 140 \mathrm{~ms}$ in men, $\geq 130 \mathrm{~ms}$ in women), C2) QS or rS pattern in the QRS complexes at leads V1 and V2 and C3) the presence of mid-QRS notch/slurs in $\geq 2$ of leads within V1, V2, V5, V6, I and aVL.

The aim of this work was to develop and assess a fullyautomatic algorithm for LBBB diagnosis based on these strict criteria, in the context of the LBBB initiative promoted by the International Society of Computerized Electrocardiology (ISCE) and the Telemetric and Holter Warehose (THEW) project [6].

\section{Study population}

Data available in this initiative are part of the Multicenter Automatic Defibrillator Implantation Trial - Cardiac Resynchronization Therapy (MADIT-CRT), conducted at University of Rochester (Rochester, NY) [7]. The original study aimed to investigate whether CRT would reduce mortality and heart failure events in patients at mild heart failure stages.

The whole dataset included 1820 randomized patients in New York Heart Association classes I and II, 1281 with LBBB, from 110 different hospital centers. Twelve-lead, high-resolution ECGs were recorded before CRT implantation using 24-hours Holter recorders (H12+, Mortara Instruments, Milwaukee, WI, USA) during 20 minutes in supine position. The study protocol was approved by each institutional review board of the participating centers.

For the present study, the organizers of the LBBB initiative only provided the ECG signals and gender label of a subset of the MADIT-CRT patients. A total of 602 10second ECG traces (sampling frequency of $1000 \mathrm{~Hz}$ and 
amplitude resolution $3.75 \mu \mathrm{V}$ ) in sinus rhythm as well as the median beat for each of the 12 leads were made available to the investigators. The dataset ( $72 \%$ men, $28 \%$ women) was divided into two sub-cohorts, conforming the training ( $n=300$ recordings) and validation datasets $(n=302$ recordings, blind $\mathrm{LBBB}$ annotations to the investigators). For the training set, global QRS duration, QRS configuration and the presence of notch/slur was delivered. Data were labeled for strict LBBB by 2 independent experts, with an additional third reviewer involved if tie-break consensus was needed. No other clinical information form the trial was made available.

\section{Methods}

According to the strict LBBB criteria, diagnosis of LBBB requires delineation of QRS boundaries, identification of QRS pattern as well as the presence of notchs/slurs within the QRS complex in selected leads. In this work we have implemented the three aforementioned conditions (C1, C2 and C3) by developing a fully automatic algorithm based on the same wavelet approach used in [8] for ECG delineation.

\subsection{Multilead QRS delineation}

First, a wavelet-based delineator [8] was applied to the median beats to detect individual $Q, R$ and $S$ waves as well as QRS boundaries $\left(Q R S_{o n}\right.$ and $\left.Q R S_{o f f}\right)$ on each standard lead.

The wavelet-based approach presented in [8] uses the derivative of a smoothing function as a prototype wavelet. Therefore, the detail coefficients can be identified as the derivative of the low-pass filtered signal at different scales. Thus, the processing acts as differenciator filter-bank. We refer the reader to [8] for a detailed description of the algorithm and its implementation.

In this wavelet decomposition of the ECG signal, the most significant components of the QRS are contained in scales $2^{1}$ to $2^{4}$. ECG wave peaks correspond to a zerocrossing at scale $2^{1}$ between a pair of maximum moduli with opposite sign at scale $2^{2}$ that exceed a fixed amplitude threshold. Negative deflections, such as Q and S waves, appear between a negative minimum-positive maximum pair, whereas positive deflections, such as R or R' waves, are identified between a positive maximum-negative minimum pair at scale $2^{2}$. The algorithm included some rules and protections based on time and sign conditions to reject deflections not defining a QRS wave (such as notches/slurs or noise artifacts).

In brief, QRS boundaries were identified before the first and after the last significant slope of the QRS, corresponding to peaks in scale $2^{2}$. $Q R S_{\text {on }}$ and $Q R S_{\text {off }}$ marks were set as the first instant for which signal amplitude at scale $2^{2}\left(x_{\mathrm{WT} 2}\right)$ was lower than two thresholds proportional to the amplitude at both maximum slopes. Temporal protections were included in order to avoid detections too distant from QRS mark.

From those single-lead annotations, multilead QRS boundaries were computed. This multilead detection was based on post-processing selection rules applied over all single-lead marks, thus providing a more robust delineation. Post-processing rules for boundaries consisted of ordering all 12 single-lead marks and setting the onset of the QRS complex $\left(Q R S_{\text {on }}^{\text {multi }}\right)$ as the earliest mark, with at least $k=2$ nearest neighbors within a $\delta=10 \mathrm{~ms}$ interval. In the same way, the end of the QRS complex $\left(Q R S_{\text {off }}^{\text {multi }}\right)$ was set as the latest annotation mark with $k=2$ nearest marks in a $\delta \mathrm{ms}$ interval. Finally, global QRS duration $\left(Q R S_{d}\right)$ for each patient was computed as the difference between $Q R S_{\text {off } f}^{\text {multi }}$ and $Q R S_{o n}^{\text {multi }}$ positions.

\subsection{QRS morphology}

The second condition for LBBB diagnosis requires QS or rS configurations (Fig. 1) in leads V1 and V2, in contrast to normal left-to-right activation of the septum, which is associated with the presence of a $\mathrm{R}$ wave in those leads.
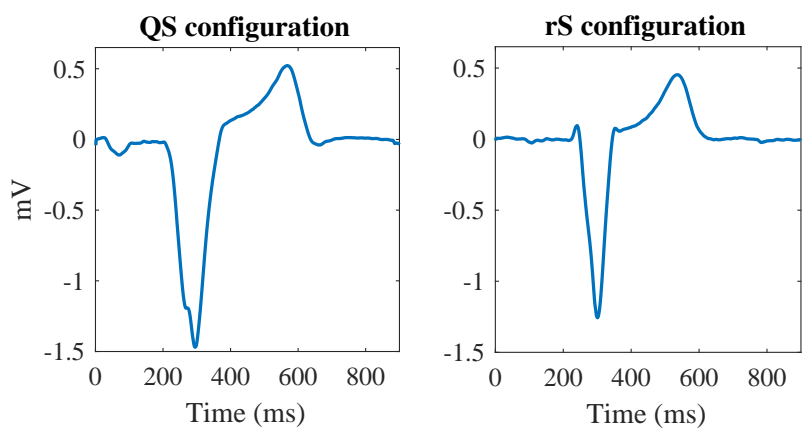

Figure 1. Illustration of $\mathrm{QR}$ and $\mathrm{rS}$ configurations of $\mathrm{QRS}$ complexes for LBBB diagnosis.

After identification of the QRS position as the main wave within the QRS complex, the wavelet-based algorithm searches for individual $\mathrm{Q}, \mathrm{R}, \mathrm{S}$ waves, considering any possible QRS configuration (QRS, RSR', RS, R, QR or QS) [8].

- A QS configuration means that only a negative deflection is detected, which corresponds to the $S$ wave, identified as the main wave of the complex. No R mark is detected.

- An rS configuration also requires the main wave to be a negative deflection, corresponding to the $S$ wave, but in this case preceded by a positive deflection of lower amplitude respect to the isoelectric line, denoted as the $\mathrm{r}$ wave. No R' wave is detected after the $\mathrm{S}$ wave. 


\subsection{Notch and slur detection}

Finally, an automatic algorithm for notch/slur detection within the QRS complex was applied based on the same wavelet-based multiscale approach used for QRS delineation.

Any peak/nadir on the ECG signal corresponds to a zero-crossing at scale $2^{1}$. We denoted those zero-crossings as $z_{i}, i=\{1, \ldots, I\}$, with $I$ the total number of detected zeros at this scale.

Any zero $z_{i}$ corresponds to a notch present in the QRS $\left(Q R S_{\text {notch }}\right)$ if there is no change in polarity on the ECG signal between $z_{i-1}$ and $z_{i+1}$, that is:

$$
\operatorname{sign}\left(x_{\mathrm{ECG}}\left(z_{i-1}\right)\right)=\operatorname{sign}\left(x_{\mathrm{ECG}}\left(z_{i}\right)\right)=\operatorname{sign}\left(x_{\mathrm{ECG}}\left(z_{i+1}\right)\right)
$$

where $x_{\mathrm{ECG}}$ denotes the ECG signal.

In addition, a minimum amplitude difference of $\gamma_{\text {notch }}=1 \mu \mathrm{V}$ between $x_{\mathrm{ECG}}\left(z_{i-1}\right)$ and $x_{\mathrm{ECG}}\left(z_{i}\right)$ and between $x_{\mathrm{ECG}}\left(z_{i}\right)$ and $x_{\mathrm{ECG}}\left(z_{i+1}\right)$ was set to consider the notch as significant. Boundaries of $Q R S_{\text {notch }}$ correspond to $z_{i-1}$ and $z_{i+1}$ for onset and offset, respectively (see Fig. 2, left column for illustration).

A slur appears as a notch on the first scale of the wavelet transform signal $\left(x_{\mathrm{WT} 1}\right)$. Thus, the algorithm is based in the same strategy but applied to this derivative signal (Fig. 2 , right column). In this case, after detection of all zerocrossings $\left(z_{j}^{\prime}, j=\{1, \ldots, J\}\right)$ on the derivative of $x_{\mathrm{wT} 1}$ within the QRS complex $\left(x_{\mathrm{wT} 1}^{\prime}\right)$, slur boundaries are identified as $z_{j-1}^{\prime}$ and $z_{j+1}^{\prime}$ for any $j$ that fulfills the following:

$\operatorname{sign}\left(x_{\mathrm{wT} 1}\left(z_{j-1}^{\prime}\right)\right)=\operatorname{sign}\left(x_{\mathrm{wT} 1}\left(z_{j}^{\prime}\right)\right)=\operatorname{sign}\left(x_{\mathrm{wT} 1}\left(z_{j+1}^{\prime}\right)\right)$.

Notch/slurs had to appear $40 \mathrm{~ms}$ after the QRS onset and before $50 \%$ of the global QRS duration to be associated to LBBB. In addition, they have to be present in at least 2 of leads V1, V2, V5, V6, I and aVL. Initially, strict LBBB criteria given by the organizers included an additional condition, requiring the notch/slur to end before 2/3 of the QRS duration. However, after ISCE's annual conference (April 2018) this condition was excluded.

\subsection{Parameter adjustment}

The whole data set was divided into a training and a validations set by the organizers. At time of submission, only sex information was made available for the validation set, while QRS boundaries and duration, presence of QS o rS configurations in V1 and V2 as well as notch/slur annotations in selected leads were available for the whole training set. This information was used for learning purposes. In particular, it was used to adjust some parameters of the wavelet-based delineator for the determination of QRS and notch/slurs boundaries. After ISCE's annual conference, organizers of the initiative made available the
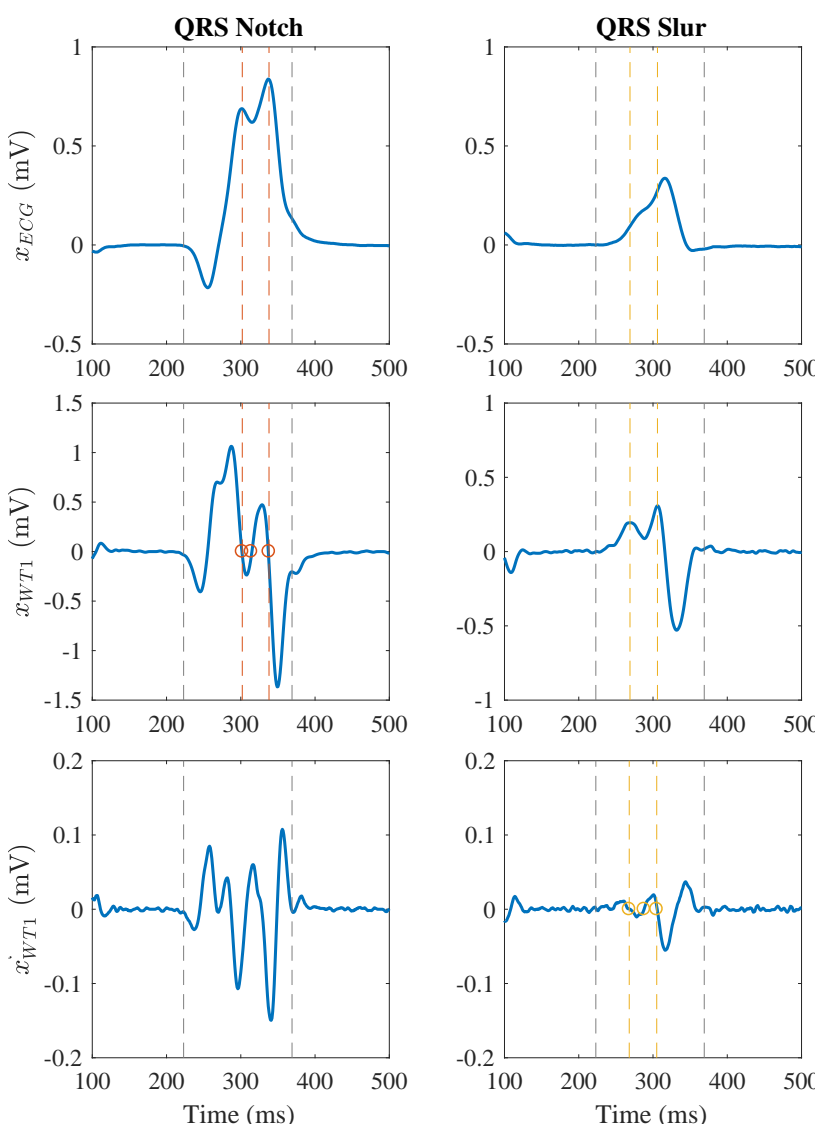

Figure 2. Illustration of notch (left) and slur (right) detection on the ECG signal based on zero-crossing on WT1 and its derivative. QRS, notch and slur boundaries are indicated by grey, red and yellow vertical lines, respectively.

corrected annotations from both training and test datasets to all participants.

\section{4. $\quad$ Results}

Final results obtained on the validation set with this new criteria are included in Table 1. It includes both intermediate results for each individual condition ( $\mathrm{C} 1$ and $\mathrm{C} 2)$, and final LBBB diagnosis.

Table 1. Automatic LBBB diagnosis using wavelet-based approach in validation set $(n=302)$.

\begin{tabular}{lccccc}
\hline \hline & Acc $(\%)$ & Se $(\%)$ & Sp(\%) & PPV(\%) & NPV(\%) \\
\hline $\mathrm{C} 1\left(Q R S_{d}\right)$ & 82.1 & 97.3 & 42.2 & 81.9 & 85.4 \\
$\mathrm{C} 2(\mathrm{QRS}$ config) & 96.0 & 98.3 & 86.9 & 96.7 & 93.0 \\
\hline LBBB diagnosis & 79.5 & 92.9 & 65.0 & 74.0 & 89.6 \\
\hline \hline
\end{tabular}

QRS configurations were accurately identified using the wavelet-based algorithm (Acc $=96.03 \%$, only 12 out of 302 cases were misclassified). On average, our algorithm over- 
estimated the $Q R S d$ with respect to manual annotations (automatic measurement: $160.5 \pm 23.5 \mathrm{~ms}$ vs manual annotations: $150.5 \pm 21.8 \mathrm{~ms}$ ), getting a significant number of false positives, which explains the low specificity rate $(42.17 \%)$ obtained when evaluating C1. Some examples are shown on Fig. 3.
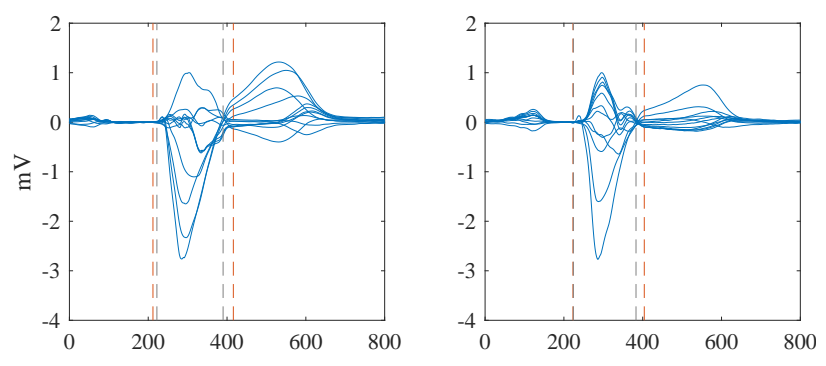

Figure 3. Buttefly plots of two of the highest discrepancies between QRS boundaries obtained from automatic (red lines) and manual annotations (grey lines).

The discrepancies in C3, i.e., the presence of mid-QRS notches/slurs between the automatic algorithm and annotations could not be quantified since experts' annotations of notches/slurs were not provided when one of the two previous conditions, $\mathrm{C} 1$ or $\mathrm{C} 2$, were not fulfilled.

Altogether, LBBB in the test dataset was diagnosed with a $79.45 \%$ of accuracy and a sensitivity and specificity rates of $\mathrm{Se}=92.95 \%$ and $\mathrm{Sp}=65.73 \%$ using the proposed automatic algorithm.

\section{Discussion and conclusion}

In the context of the LBBB initiative, we have developed a fully-automatic algorithm for strict LBBB diagnosis. The algorithm uses the wavelet transformation approach implemented in [8] for detection/delineation of ECG waves. After identification of main waves and QRS boundaries, the algorithm takes the advantage of the wavelet transformation for the delineation of notches/slurs boundaries, step that was not present in the original algorithm.

The first condition for LBBB diagnosis requires prolonged QRS complex. Our multilead approach for computation of global QRS duration tended to overestimate this measurement, and we observed that these differences were mainly due to a delayed position of the $Q R S_{\text {off } f}$ mark respect to manual annotations (based on a butterfly plot). More than a half of misdiagnosed LBBB (38 out of 62) were attributable to disagreements on this condition.

More accurate results were obtained in the identification of QS and rS configurations in leads V1 and V2. Indeed, we observed that 6 out of the 8 false positives obtained in the evaluation set were due to an $\mathrm{rS}$ decision, whereas it seemed that they were consider as RS by the experts. Our initial condition required the absolute amplitude of $\mathrm{R}$ wave respect to the isoelectric line to be lower than $\mathrm{S}$ wave amplitude, (i.e, R/S <1), however experts required a lower ratio to consider an $\mathrm{rS}$ pattern.

The final condition, and the most challenging one, was the detection of mid-QRS notches or slurs in some leads. Notches/slurs were not annotated in all recordings, thus limiting the statistical analysis in our results. Nevertheless, only 19 out of 62 final inaccurate LBBB diagnosis were due to discrepancies in this third condition.

In conclusion, results confirmed an accurate diagnosis of strict LBBB based on a fully-automatic extraction of temporal and morphological QRS features.

\section{Acknowledgements}

This work was supported by CIBER in Bioengineering, Biomaterials \& Nanomedicne (CIBER-BBN) through Instituto de Salud Carlos III and FEDER (Spain), project DPI2016-75458-R funded by MINECO and FEDER, and by Gobierno de Aragón and European Social Fund (EU) through BSICoS Reference Group (T39-17R). The computation was performed by the ICTS NANBIOSIS, specifically by the High Performance Computing Unit of the CIBER-BBN at the University of Zaragoza.

\section{References}

[1] Zareba et al. Effectiveness of cardiac resynchronization therapy by QRS morphology in the multicenter automatic defibrillator implantation trial Cardiac resynchronization therapy (MADIT-CRT). Circulation 2011;123:1061-1072

[2] Mascioli G1 et al. Electrocardiographic criteria of true left bundle branch block: a simple sign to predict a better clinical and instrumental response to CRT. Pacing and clinical electrophysiology 2012;35:927-924

[3] Grant RP, Dodge HT. Mechanisms of QRS complex prolongation in man: Left ventricular conduction disturbances. The American Journal of Medicine 1956;20:834-852

[4] Auricchio et al. Characterization of left ventricular activation in patients with heart failure and left bundle-branch block. Circulation 2004;109:1133-1139

[5] Strauss DG, Selvester RH, Wagner GS. Defining left bundle branch block in the era of cardiac resynchronization therapy. The American Journal of Cardiology 2011;107(6):927-934

[6] Guidelines for the LBBB Initiative of the ISCE 2018 meeting http://thew-project.org/LBBB_Initiative.htm

[7] Moss AJ et al. Cardiac-resynchronization therapy for the prevention of heart-failure events. The New England Journal of Medicine, 2009;361:1329-1338

[8] Martínez JP et al. Wavelet-based ECG delineator: evaluation on standard databases. IEEE T Biomed Eng 2004;51:570-581

Address for correspondence:

Alba Martín Yebra, Universidad de Zaragoza

C/ María de Luna, 1, Ed. Ada Byron, Lab. 3.07

50018, Zaragoza, Spain

e-mail: amartiny@unizar.es 